Investigación descriptiva, correlacional o cualitativa

Pensar en Movimiento:

Revista de Ciencias del Ejercicio y la Salud

EISSN: 1659-4436

Vol. 13, № 2, pp. 17- 33

Cierre al 31 de diciembre, 2015

\title{
DIFERENCIAS EN EL DESEMPEÑO DE LOS PATRONES BÁSICOS DE MOVIMIENTO SEGÚN LA EDAD Y EL SEXO
}

(versión traducida al español) ${ }^{1}$

\author{
Judith Jiménez-Díaz, M. Sc. ${ }^{a(A, B, C, D, E)}$, Walter Salazar-Rojas, Ph. D. ${ }^{a(B, D, E)}$ \\ y María Morera, Ph. D.b(B,D,E) \\ judith.jimenezd@ucr.ac.cr \\ aEscuela de Educación Física y Deportes, Universidad de Costa Rica, \\ San José, Costa Rica \\ ${ }^{b}$ Escuela de Movimiento Humano y Calidad de Vida, Universidad Nacional, \\ Heredia, Costa Rica
}

Envío: 04-03-2015 Reenvíos: 03-04-2015/11-07-2015/19-08-2015

Aceptado: 27/08/2015. Publicado: 25/11/2015

doi: http://dx.doi.org/10.15517/pensarmov.v13i2.22024

\begin{abstract}
Resumen
Jiménez-Díaz, J., Salazar-Rojas, W. y Morera, M. (2015). Diferencias en el desempeño de los patrones básicos de movimiento según la edad y el sexo. Pensar en Movimiento: Revista de Ciencias del Ejercicio y la Salud, 13(2), 17-33. Los patrones básicos de movimiento son la base para participar en actividades más complejas a lo largo de la vida. Mientras que una cantidad considerable de investigaciones ha reportado el comportamiento motor en la edad infantil, existe un desconocimiento acerca de este desempeño en edades subsecuentes, especialmente en la adultez. Por lo anterior, el objetivo principal de este estudio fue examinar las diferencias de edad y sexo en del desempeño de los patrones básicos de movimiento en tres grupos de edades: infantes $(M$ $=9,37$ años de edad, $D E=1,26)$, adolescentes $(M=14,80$ años de edad, $D E=2,04)$ y adultos $(M=19,88$ años de edad, $D E=2,72)$. Un total de 114 participantes fueron evaluados en cinco patrones locomotores y cinco manipulativos con la utilización del instrumento para la evaluación de patrones básicos de movimiento, el cual es un instrumento orientado al proceso del movimiento. Los resultados del ANOVA, al comparar edad y sexo no presentaron interacción significativa. Adicionalmente, el efecto principal de grupo mostró diferencias en tres destrezas individuales: galopar-adolescentes y adultos presentaron mejor desempeño que los infantes $(p<, 01)$-, lanzar -infantes y adolescentes
\end{abstract}

\footnotetext{
${ }^{1}$ También disponible en la versión original en inglés en esta misma revista.
} 
presentaron mejor desempeño que los adultos $(p<, 01)-\mathrm{y}$ patear -adultos presentaron mejor desempeño que los infantes y adolescentes $(p<, 05)-$. Además, se encontraron diferencias significativas en el efecto principal de sexo para el total del desempeño motor $(p<, 01)$, en la subescala de patrones manipulativos $(p<, 01)$ y locomotores $(p<, 05)$, y en seis destrezas individuales: correr $(p<, 05)$, saltar $(p<, 05)$, lanzar $(p<, 01)$, patear $(p$ $<, 01)$, rebotar $(p<, 01)$ y batear $(p<, 01)$; los hombres siempre presentan un mejor desempeño que las mujeres. Por tanto, es importante fortalecer los patrones básicos de movimiento a todas las edades, como una opción para ayudar a las personas a realizar actividades físicas.

Palabras clave: locomoción, manipulación desempeño motor, adulto, diferencias de género, envejecimiento

A pesar de que se conocen los beneficios de ser físicamente activo, los índices de sedentarismo han incrementado a nivel mundial. Aunque los mecanismos que causan la inactividad física no se comprenden detalladamente (Okely, Booth, \& Patterson, 2001; Stodden et al., 2008); se cree que el desempeño motor presenta un rol importante en los hábitos de actividad física de las personas (Stodden et al., 2008).

Los patrones básicos de movimiento (PBM) son destrezas necesarias para participar en deportes, juegos y en actividades físicas a lo largo de la vida (Logan, Robinson, Wilson, \& Lucas, 2012; Stodden et al., 2008). Los PBM usualmente se clasifican en movimientos locomotores y manipulativos. Los patrones locomotores son los movimientos que implican que el cuerpo se mueva en el espacio, como correr, saltar y galopar. Los movimientos manipulativos consisten en proyectar o recibir objetos, como lanzar, apañar y batear (Gabbard, 2012; Stodden \& Goodway, 2007).

Cuando una persona sabe cómo moverse en el nivel más básico, le es más sencillo participar en diferentes actividades físicas (Carley, 2010; Stodden et al., 2008). Por lo tanto, estamos de acuerdo con que el desempeño en los PBM es un factor muy importante para tener éxito en movimientos más complejos y también, motiva a las personas a participar en juegos, actividades deportivas y recreativas, de tal manera que se promueve una vida más activa, la cual es necesaria para mantener niveles adecuados de las capacidades físicas relacionadas con la salud (Carley, 2010; Stodden, Langendorfer, \& Roberton, 2009; Valentini \& Rudisill, 2004). Por el contrario, una persona que presente un nivel bajo de desempeño de los PBM, tiene más probabilidades de ser físicamente inactivo, debido a que la falta de habilidad afecta de manera negativa la intención de participar en juegos y deportes con los amigos (as), lo que causa que no se 
obtengan niveles saludables de capacidades físicas relacionadas con la salud (Carley, 2010; Okely et al., 2001).

La evidencia sugiere que el desempeño en los PBM está relacionado positivamente con los niveles de actividad física (Cohen, Morgan, Plotnikoff, Callister, \& Lubans, 2014; Okely et al., 2001; Spessato, Gabbard, \& Valentini, 2013; Wrotniak, Epstein, Dorn, Jones, \& Kondilis, 2006) y con las capacidades físicas relacionadas con la salud (Okely, Booth, \& Chey, 2004; Slotte, Sääkslahti, Metsämuuronen, \& Rintala, 2014; Southall, Okely, \& Steele, 2004). Por ejemplo, Wrotniak et al. (2006) encontró que el desempeño en PBM en niños(as) está relacionado positivamente con el nivel de actividad. De igual manera, Okely et al. (2001) concluyeron que participar en actividades físicas organizadas está relacionado con el desempeño de los PBM en adolescentes. Además, Cohen et al. (2014) indicaron que los niños(as) con mejor desempeño dedican más tiempo a realizar actividad física moderada y vigorosa.

En relación con las capacidades físicas relacionadas a la salud, Slotte et al. (2004) encontraron que el desempeño en PBM está asociado negativamente al porcentaje de grasa corporal en infantes de 8 años de edad. De manera similar, Southall et al. (2004) reportaron una relación inversa entre el desempeño en PBM y el índice de masa corporal (IMC) en niños y niñas. Adicionalmente, Stodden et al. (2009) encontraron una asociación positiva entre el desempeño de los PBM y las capacidades físicas relacionadas con la salud, en donde se incluye fuerza y resistencia muscular, resistencia cardiovascular, y además reportaron una relación negativa entre el desempeño de PBM y porcentaje de grasa corporal en adultos-jóvenes.

Seefeldt planteó la hipótesis que indica que es necesario un nivel mínimo de desempeño en PBM para mantener un estilo de vida físicamente activo, que eventualmente va a promover un nivel saludable de las capacidades físicas relacionadas con la salud (Stodden et al., 2008). Al considerar que no hay suficiente evidencia que indica cuál es el desempeño mínimo, creemos que puede ser entendido como una etapa madura. Esta etapa se caracteriza por un desempeño controlado y comúnmente se desarrolla a los seis o siete años de edad, y se refina en los años siguientes (Gallahue \& Ozmun, 2005). En este orden de ideas, es importante resaltar que para desarrollar un patrón maduro y mejorar el desempeño de los PBM, estos deben ser aprendidos, practicados y reforzados de forma adecuada (Goodway \& Branta, 2003; Logan et al., -19 -

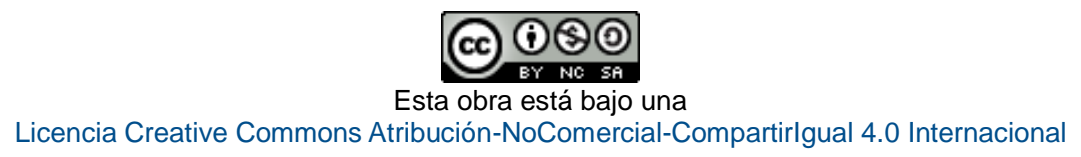


2012).

A pesar de la relevancia que presenta un desempeño eficiente de los PBM, como movimientos fundamentales, la evidencia indica que el desempeño en los niños(as) es bajo (Lubans, Morgan, Cliff, Barnett, \& Okely, 2010; Spessato, Gabbard, Valentini, \& Rudisill, 2012). Además, se conoce poco acerca del desempeño de los PBM en etapas posteriores a la niñez (Barnett, van Beurden, Morgan, Brooks, \& Beard, 2010; Logan et al., 2012). Por tanto, el objetivo del presente estudio es examinar las diferencias de edad y sexo en diez patrones básicos de movimiento, entre tres grupos de edades -infantes, adolescentes y adultos-jóvenes-.

\section{METODOLOGÍA}

Participantes. Un total de 114 participantes (ver tabla 1), hombres $(n=59)$ y mujeres $(n=55)$, con edades entre los 8 y 27 años de edad, participaron de forma voluntaria en el presente estudio. Las personas participantes con edades entre los 8 y 17 años asistieron a un programa de actividades recreativas de verano en una universidad en Costa Rica; mientras que los participantes con edades entre los 18 y 27 estaban matriculados en diferentes clases de Educación Física en la misma universidad. Todas las personas participantes se encontraban con un buen estado de salud y no presentaron impedimentos motrices. Se obtuvo el consentimiento y el asentimiento de los participantes y/o su responsable legal antes de iniciar el estudio.

Instrumentos. Se utilizó el Instrumento para la evaluación de Patrones Básicos de Movimiento -IPBM- para evaluar el desempeño en los patrones básicos de movimiento en los infantes, adolescentes y adultos (Jiménez, Salazar, \& Morera, 2013). Esta es una prueba orientada al proceso que evalúa 10 patrones básicos de movimiento, compuesto por dos subescalas: patrones locomotores (LM) (correr, galopar, deslizar, saltar a distancia y brincar) y patrones de manipulación (OC) (apañar, patear, batear, lanzar por encima del hombro y rebotar). La confiabilidad (coeficiente intraclase $R=0,918$ ) y la objetividad (coeficiente interevaluadores $R=0,861$ ) son adecuados. El puntaje neto para cada patrón de movimiento, cada subescala (LM y OC) y para el total de la prueba oscila entre los 0 y 12 puntos. El puntaje total para cada subescala es un promedio de los cinco patrones 
correspondientes a la subescala. Igualmente, el puntaje total de la prueba es un promedio de los 10 patrones. Un puntaje igual o mayor a 9 puntos indica un desempeño eficiente de los PBM.

La talla $(\mathrm{cm})$ se midió con una tabla portátil. Se utilizó una balanza de bioimpedancia (BF-682W TANITA) para obtener el peso $(\mathrm{Kg})$. El peso y la talla se obtuvieron con fines descriptivos de la muestra.

Procedimientos. Siguiendo los procedimientos institucionales de investigación, todas las personas fueron pesadas y medidas, después de firmar el consentimiento informado y haber contestado un cuestionario de información personal. Para obtener el desempeño de los PBM, se filmó a los participantes ejecutando los diez PBM (correr, galopar, deslizar, saltar a distancia, brincar, apañar, patear, batear, lanzar por encima del hombro y rebotar) en dos intentos. La medición se llevó a cabo en un gimnasio, en donde se evaluó un patrón a la vez y un participante a la vez. El desempeño se evaluó de la filmación. Como se indica en el manual de la prueba, los participantes reciben 1 punto por cada criterio presente y 0 puntos si no está presente (Jiménez et al., 2013). El puntaje total por subescala y para el total de la prueba se calculó de la forma indicada previamente.

Tabla 1

Características de los participantes

\begin{tabular}{|c|c|c|c|c|}
\hline \multirow{2}{*}{ Grupo } & \multirow{2}{*}{ Características } & \multicolumn{3}{|c|}{$\underline{\text { Sexo }}$} \\
\hline & & Masculino & Femenino & Total \\
\hline & $\mathrm{N}$ & 22 & 16 & 38 \\
\hline Infantes & Edad (años) & $9,64(1,29)$ & $9,00(1,15)$ & $9,37(1,26)$ \\
\hline \multirow[t]{3}{*}{ (8-11 años) } & Peso $(\mathrm{kg})$ & $36,19(8,55)$ & $31,23(5,33)$ & $34,10(7,69)$ \\
\hline & Talla (cm) & $138,75(8,27)$ & $132,46(8,64)$ & $136,10(8,89)$ \\
\hline & $\mathrm{N}$ & 18 & 17 & 35 \\
\hline \multirow{4}{*}{$\begin{array}{l}\text { Adolescentes } \\
\text { (12-17 años) }\end{array}$} & Edad (años) & $14,33(2,09)$ & $15,29(1,93)$ & $14,80(2,04)$ \\
\hline & Peso (kg) & $63,33(14,56)$ & $57,64(12,24)$ & $60,57(13,60)$ \\
\hline & Talla (cm) & $162,72(12,75)$ & $157,41(7,19)$ & $160,14(10,63)$ \\
\hline & $\mathrm{N}$ & 19 & 22 & 41 \\
\hline \multirow{3}{*}{$\begin{array}{l}\text { Adultos-jóvenes } \\
\text { (18-27 años) }\end{array}$} & Edad (años) & $19,42(1,98)$ & $20,27(3,22)$ & $19,88(2,72)$ \\
\hline & Peso $(\mathrm{kg})$ & $62,53(10,42)$ & $59,90(10,86)$ & $61,12(10,61)$ \\
\hline & Talla (cm) & $170,84(5,62)$ & $160,50(5,46)$ & $165,29(7,56)$ \\
\hline
\end{tabular}

Los datos se presentan en Promedio (Desviación Estándar). Fuente: elaboración propia. 
Análisis de los datos. Se analizó la distribución normal de los datos (Gráfico Q-Q y prueba de Levene). Se calculó el rango, medias, desviaciones estándar y frecuencias con fines descriptivos. Se aplicó un ANOVA de dos vías (Grupo [3] x Sexo [2]) para examinar las diferencias en el desempeño de los PBM (para el puntaje total, el puntaje por subescala y el puntaje de cada patrón). Se utilizó la prueba de post-hoc de Tuckey. Todos los análisis se realizaron con el programa IBM-SPSS® statistics 21 . Se usó un nivel de alfa de 0,05 .

\section{RESULTADOS}

Los gráficos de residuos y la prueba de normalidad indicaron que los datos presentan una distribución normal. Los puntajes obtenidos para los 10 patrones, las subescalas y el puntaje total de la prueba se muestran en la tabla 2 . El $36,8 \%$ de los niños y niñas, el 45,7 \% de los adolescentes y el 36,5 \% de los adultos-jóvenes evaluados obtuvieron un nivel eficiente de desempeño (ver figura 1). En la tabla 3 se muestra el porcentaje de personas que alcanzaron un nivel de desempeño eficiente para los PBM, las subescalas y el total de la prueba. Los resultados de ANOVA no muestran interacción significativa para el puntaje total, las subescalas de LM y OC y ninguno de los PBM. Se encontró efecto principal significativo para grupo y sexo. Los resultados de ANOVA se presentan en la tabla 4.

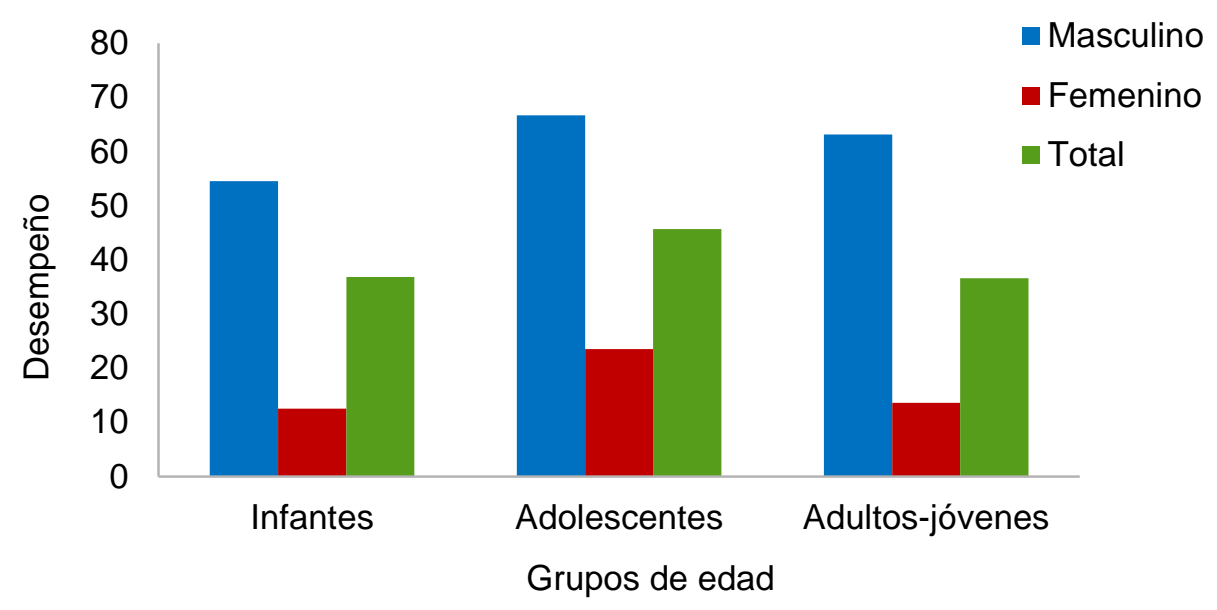

Figura 1. Porcentaje de participantes que presentaron desempeño eficiente en el desempeño de los Patrones Básicos de Movimiento. Fuente: elaboración propia. 
Tabla 2

Estadística descriptiva de los participantes en el desempeño de los PBM

\begin{tabular}{|c|c|c|c|c|c|c|c|c|c|}
\hline \multirow{2}{*}{ PBM } & \multicolumn{3}{|c|}{$\underline{\text { Infantes }}$} & \multicolumn{3}{|c|}{$\underline{\text { Adolescentes }}$} & \multicolumn{3}{|c|}{$\underline{\text { Adultos-jóvenes }}$} \\
\hline & Masc. & Fem. & Total & Masc. & Fem. & Total & Masc. & Fem. & Total \\
\hline Correr & $\begin{array}{l}9,00 \\
(1,8)\end{array}$ & $\begin{array}{l}8.69 \\
(1,2)\end{array}$ & $\begin{array}{l}8,87 \\
(1,6)\end{array}$ & $\begin{array}{r}9,06 \\
(1,8)\end{array}$ & $\begin{array}{r}8,12 \\
(1,4)\end{array}$ & $\begin{array}{l}8,60 \\
(1,6)\end{array}$ & $\begin{array}{r}9,37 \\
(1,3)\end{array}$ & $\begin{array}{r}8,64 \\
(1,8)\end{array}$ & $\begin{array}{r}8,98 \\
(1,6)\end{array}$ \\
\hline Galopar & $\begin{array}{l}7,86 \\
(2,6)\end{array}$ & $\begin{array}{l}6,94 \\
(3,8)\end{array}$ & $\begin{array}{l}7,47 \\
(3,1)\end{array}$ & $\begin{array}{l}8,94 \\
(2,9)\end{array}$ & $\begin{array}{l}8,29 \\
(2,2)\end{array}$ & $\begin{array}{l}8,63 \\
(2,5)\end{array}$ & $\begin{array}{r}10,05 \\
(1,6)\end{array}$ & $\begin{array}{l}8,86 \\
(1,5)\end{array}$ & $\begin{array}{l}9,41 \\
(1,6)\end{array}$ \\
\hline Brincar & $\begin{array}{l}9,00 \\
(1,9)\end{array}$ & $\begin{array}{l}8,25 \\
(1,9)\end{array}$ & $\begin{array}{l}8,68 \\
(1,9)\end{array}$ & $\begin{array}{l}8,72 \\
(2,8)\end{array}$ & $\begin{array}{l}8,29 \\
(1,7)\end{array}$ & $\begin{array}{l}8,51 \\
(2,3)\end{array}$ & $\begin{array}{l}8,79 \\
(2,3)\end{array}$ & $\begin{array}{l}8,64 \\
(1,8)\end{array}$ & $\begin{array}{l}8,71 \\
(2,0)\end{array}$ \\
\hline Saltar & $\begin{array}{l}7,41 \\
(29)\end{array}$ & $\begin{array}{l}7,06 \\
(2,9)\end{array}$ & $\begin{array}{l}7,26 \\
(2,9)\end{array}$ & $\begin{array}{l}9,44 \\
(2,6)\end{array}$ & $\begin{array}{l}7,59 \\
(2,8)\end{array}$ & $\begin{array}{l}8,54 \\
(2,8)\end{array}$ & $\begin{array}{l}9,32 \\
(2,5)\end{array}$ & $\begin{array}{l}7,82 \\
(2,6)\end{array}$ & $\begin{array}{l}8,51 \\
(2,6)\end{array}$ \\
\hline Deslizar & $\begin{array}{l}8,27 \\
(2,4)\end{array}$ & $\begin{array}{l}8,56 \\
(2,5)\end{array}$ & $\begin{array}{l}8,39 \\
(2,4)\end{array}$ & $\begin{array}{l}8,33 \\
(2,6)\end{array}$ & $\begin{array}{l}9,00 \\
(1,4)\end{array}$ & $\begin{array}{l}8,66 \\
(2,1)\end{array}$ & $\begin{array}{l}8,26 \\
(2,1)\end{array}$ & $\begin{array}{l}8,05 \\
(2,5)\end{array}$ & $\begin{array}{l}8,15 \\
(2,3)\end{array}$ \\
\hline Rebotar & $\begin{array}{l}7,68 \\
(1,8)\end{array}$ & $\begin{array}{l}6,63 \\
(2,4)\end{array}$ & $\begin{array}{l}7,24 \\
(2,1)\end{array}$ & $\begin{array}{l}8,44 \\
(1,7)\end{array}$ & $\begin{array}{l}6,82 \\
(2,5)\end{array}$ & $\begin{array}{l}7,66 \\
(2,2)\end{array}$ & $\begin{array}{l}8,84 \\
(1,5)\end{array}$ & $\begin{array}{l}7,18 \\
(1,5)\end{array}$ & $\begin{array}{l}7,95 \\
(1,7)\end{array}$ \\
\hline Lanzar & $\begin{array}{l}8,55 \\
(3,1)\end{array}$ & $\begin{array}{l}6,50 \\
(3,5)\end{array}$ & $\begin{array}{l}7,68 \\
(3,4)\end{array}$ & $\begin{array}{l}9,11 \\
(2,1)\end{array}$ & $\begin{array}{l}6,65 \\
(4,0)\end{array}$ & $\begin{array}{l}7,91 \\
(3,4)\end{array}$ & $\begin{array}{l}5,26 \\
(2,6)\end{array}$ & $\begin{array}{l}4,64 \\
(3,0)\end{array}$ & $\begin{array}{l}4,93 \\
(2,8)\end{array}$ \\
\hline Apañar & $\begin{array}{l}9,50 \\
(2,1)\end{array}$ & $\begin{array}{l}9,94 \\
(1,8)\end{array}$ & $\begin{array}{l}9,68 \\
(1,9)\end{array}$ & $\begin{array}{l}9,06 \\
(1,7)\end{array}$ & $\begin{array}{l}8,94 \\
(1,8)\end{array}$ & $\begin{array}{l}9,00 \\
(1,7)\end{array}$ & $\begin{array}{l}9,21 \\
(1,7)\end{array}$ & $\begin{array}{l}8,82 \\
(1,9)\end{array}$ & $\begin{array}{l}9,00 \\
(1,8)\end{array}$ \\
\hline Patear & $\begin{array}{r}10,73 \\
(2,1)\end{array}$ & $\begin{array}{l}7,50 \\
(2,3)\end{array}$ & $\begin{array}{l}9,37 \\
(2,7)\end{array}$ & $\begin{array}{r}10,28 \\
(2,6)\end{array}$ & $\begin{array}{l}8,41 \\
(2,4)\end{array}$ & $\begin{array}{l}9,37 \\
(2,6)\end{array}$ & $\begin{array}{r}11,47 \\
(0,8)\end{array}$ & $\begin{array}{l}9,23 \\
(1,6)\end{array}$ & $\begin{array}{r}10,27 \\
(1,7)\end{array}$ \\
\hline Batear & $\begin{array}{l}9,55 \\
(2,2)\end{array}$ & $\begin{array}{l}8,31 \\
(2,4)\end{array}$ & $\begin{array}{l}9,03 \\
(2,3)\end{array}$ & $\begin{array}{l}9,06 \\
(1,5)\end{array}$ & $\begin{array}{l}7,59 \\
(1,9)\end{array}$ & $\begin{array}{l}8,34 \\
(1,9)\end{array}$ & $\begin{array}{l}9,89 \\
(1,7)\end{array}$ & $\begin{array}{l}7,41 \\
(2,4)\end{array}$ & $\begin{array}{l}8,56 \\
(2,4)\end{array}$ \\
\hline LM & $\begin{array}{l}8,31 \\
(1,2)\end{array}$ & $\begin{array}{l}7,90 \\
(1,2)\end{array}$ & $\begin{array}{l}8,14 \\
(1,2)\end{array}$ & $\begin{array}{l}8,90 \\
(2,1)\end{array}$ & $\begin{array}{l}8,26 \\
(1,0)\end{array}$ & $\begin{array}{l}8,59 \\
(1,7)\end{array}$ & $\begin{array}{l}9,16 \\
(1,0)\end{array}$ & $\begin{array}{l}8,40 \\
(1,1)\end{array}$ & $\begin{array}{l}8,75 \\
(1,1)\end{array}$ \\
\hline OC & $\begin{array}{l}9,20 \\
(1,7)\end{array}$ & $\begin{array}{l}7,78 \\
(1,4)\end{array}$ & $\begin{array}{l}8,60 \\
(1,7)\end{array}$ & $\begin{array}{l}9,19 \\
(1,1)\end{array}$ & $\begin{array}{r}7,68 \\
(1,8)\end{array}$ & $\begin{array}{l}8,46 \\
(1,6)\end{array}$ & $\begin{array}{l}8,94 \\
(0,9)\end{array}$ & $\begin{array}{l}7,45 \\
(1,1)\end{array}$ & $\begin{array}{l}8,14 \\
(1,3)\end{array}$ \\
\hline IPBM & $\begin{array}{l}8,75 \\
(1,2)\end{array}$ & $\begin{array}{l}7,84 \\
(0,9)\end{array}$ & $\begin{array}{l}8,37 \\
(1,2)\end{array}$ & $\begin{array}{l}9,04 \\
(1,4)\end{array}$ & $\begin{array}{l}7,97 \\
(1,2)\end{array}$ & $\begin{array}{l}8,52 \\
(1,4)\end{array}$ & $\begin{array}{l}9,05 \\
(0,6)\end{array}$ & $\begin{array}{r}7,93 \\
(0,9)\end{array}$ & $\begin{array}{l}8,45 \\
(1,0)\end{array}$ \\
\hline
\end{tabular}

Los datos se presentan en Promedio (Desviación Estándar). Fuente: elaboración propia.

\section{Diferencias en grupos de edades}

No se encontraron diferencias para el puntaje total ni para las subescalas (ver figura 2), lo que indica que los tres grupos de edades presentan un desempeño similar. En relación con los patrones individuales, se encontró diferencias entre grupos para el patrón de galopar (los adultos-jóvenes y los adolescentes mostraron un mejor desempeño que los infantes), lanzar (los adultos-jóvenes presentaron un menor desempeño que los infantes y los adolescentes) y patear (los adultos-jóvenes presentaron un mejor desempeño que los infantes y los adolescentes) (ver figura 3). 
Tabla 3

Porcentaje de participantes que presentan un desempeño eficiente en el IPBM

\begin{tabular}{|c|c|c|c|c|c|c|c|c|c|}
\hline \multirow{2}{*}{ FMS } & \multicolumn{3}{|c|}{$\underline{\text { Infantes }}$} & \multicolumn{3}{|c|}{$\underline{\text { Adolescentes }}$} & \multicolumn{3}{|c|}{ Adultos-ióvenes } \\
\hline & Masc. & Fem. & Total & Masc. & Fem. & Total & Masc. & Fem. & Total \\
\hline Correr & 59,1 & 56,3 & 57,9 & 61,1 & 35,3 & 48,6 & 73,7 & 59,1 & 65,9 \\
\hline Galopar & 45,5 & 37,5 & 42,1 & 66,7 & 64,7 & 65,7 & 89,5 & 59,1 & 73,2 \\
\hline Brincar & 63,6 & 37,5 & 52,6 & 61,1 & 52,9 & 57,1 & 63,2 & 54,5 & 58,5 \\
\hline Saltar & 40,9 & 37,5 & 39,5 & 61,1 & 41,2 & 51,4 & 68,4 & 40,9 & 53,7 \\
\hline Deslizar & 40,9 & 62,5 & 50,0 & 55,6 & 70,6 & 62,9 & 36,8 & 40,9 & 39,0 \\
\hline Rebotar & 27,3 & 12,5 & 21,1 & 44,4 & 23,5 & 34,3 & 42,1 & 13,6 & 26,8 \\
\hline Lanzar & 54,5 & 25,0 & 42,1 & 50,0 & 47,1 & 48,6 & 15,8 & 18,2 & 17,1 \\
\hline Apañar & 77,3 & 75,0 & 76,3 & 61,1 & 64,7 & 62,9 & 63,2 & 54,5 & 58,5 \\
\hline Patear & 86,4 & 37,5 & 65,8 & 83,3 & 47,1 & 65,7 & 100,0 & 68,2 & 82,9 \\
\hline Batear & 77,3 & 56,3 & 68,4 & 61,1 & 35,3 & 48,6 & 78,9 & 40,9 & 58,5 \\
\hline LM & 31,8 & 18,8 & 26,3 & 72,2 & 17,6 & 45,7 & 52,6 & 22,7 & 36,6 \\
\hline OC & 63,6 & 25,0 & 47,4 & 61,1 & 35,3 & 48,6 & 42,1 & 4,5 & 22,0 \\
\hline Total IPBM & 54,5 & 12,5 & 36,8 & 66,7 & 23,5 & 45,7 & 63,2 & 13,6 & 36,6 \\
\hline
\end{tabular}

Datos se presentan en porcentajes. Fuente: elaboración propia.

Tabla 4

Valores F de ANOVA para los efectos principales y la interacción

\begin{tabular}{lcccccc}
\hline & \multicolumn{2}{c}{ Grupo } & \multicolumn{2}{c}{ Sexo } & \multicolumn{2}{c}{ Grupo x Sexo } \\
\cline { 2 - 7 } \multicolumn{1}{c}{ PBM } & \multicolumn{1}{c}{$F$} & $p$ & $F$ & $p$ & $F$ & $p$ \\
\hline Correr &, 634 &, 533 & $4,737^{*}$ &, 032 &, 357 &, 701 \\
Galopar & $6,687^{*}$ &, 002 & 3,862 &, 052 &, 111 &, 895 \\
Brincar &, 089 &, 915 & 1,243 &, 267 &, 194 &, 824 \\
Saltar & 2,904 &, 059 & $5,826^{*}$ &, 017 &, 776 &, 463 \\
Deslizar &, 472 &, 625 &, 324 &, 570 &, 356 &, 701 \\
Rebotar & 1,978 &, 143 & $16,212^{*}$ &, 001 &, 295 &, 745 \\
Lanzar & $10,354^{*}$ &, 001 & $8,584^{*}$ &, 004 &, 934 &, 396 \\
Apañar & 1,827 &, 166 &, 004 &, 948 &, 496 &, 610 \\
Patear & $4,079^{*}$ &, 020 & $40,468^{*}$ &, 001 & 1,083 &, 342 \\
Batear &, 774 &, 464 & $19,576^{*}$ &, 001 & 1,013 &, 366 \\
Subescala LM & 2,63 &, 076 & $5,86^{*}$ &, 017 &, 174 &, 841 \\
Subescala OC &, 503 &, 606 & $32,21^{*}$ &, 001 &, 008 &, 992 \\
Total IPBM &, 428 &, 653 & $25,641^{*}$ &, 001 &, 091 &, 913 \\
\hline
\end{tabular}

${ }^{\star} p<, 05$. Fuente: elaboración propia. 


\section{Diferencias de sexo}

Se encontró diferencias de sexo para el puntaje total y para ambas subescalas, lo que indica que los hombres presentaron un mejor desempeño que las mujeres (ver figura 4). Se encontró que los hombres mostraron un mejor desempeño en los patrones básicos de correr, saltar, rebotar, lanzar, patear y batear, con respecto a las mujeres (ver figura 5).

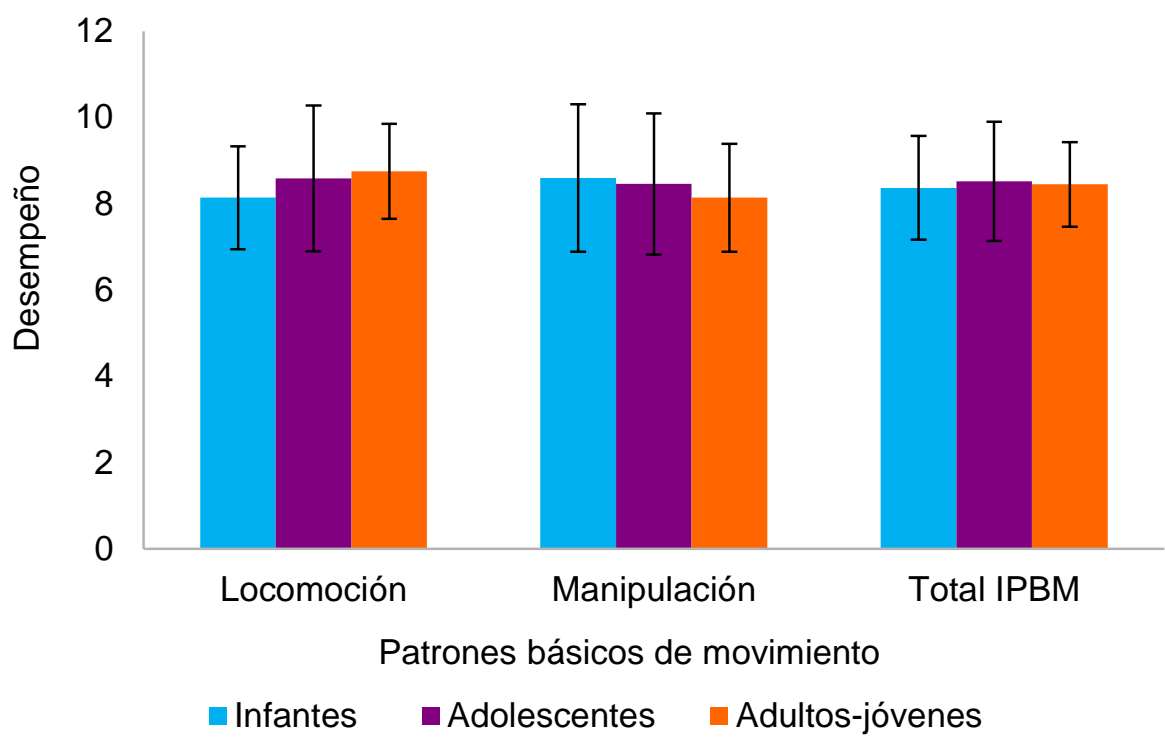

Figura 2. Desempeño total de los patrones básicos de movimiento, por subescalas según cada grupo de edad. Fuente: elaboración propia. 


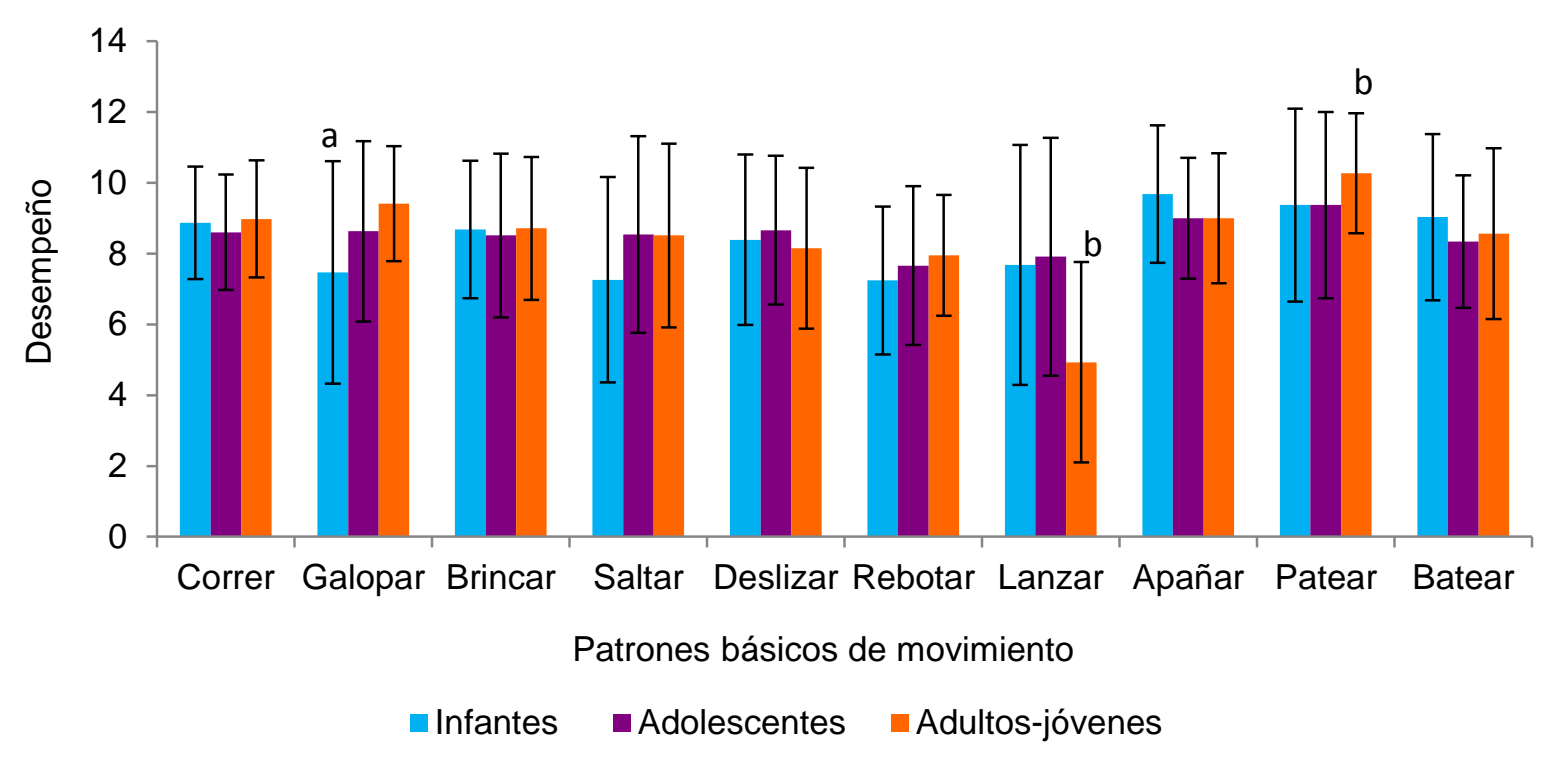

Figura 3. Desempeño de los patrones básicos de movimiento según cada grupo de edad. a El desempeño de los niños(as) es menor que los adolescente y adultos en galopar. ${ }^{\mathrm{b}} \mathrm{El}$ desempeño de los adultos-jóvenes es diferente de los infantes y adolescentes en lanzar y patear $(p<, 05)$. Fuente: elaboración propia.

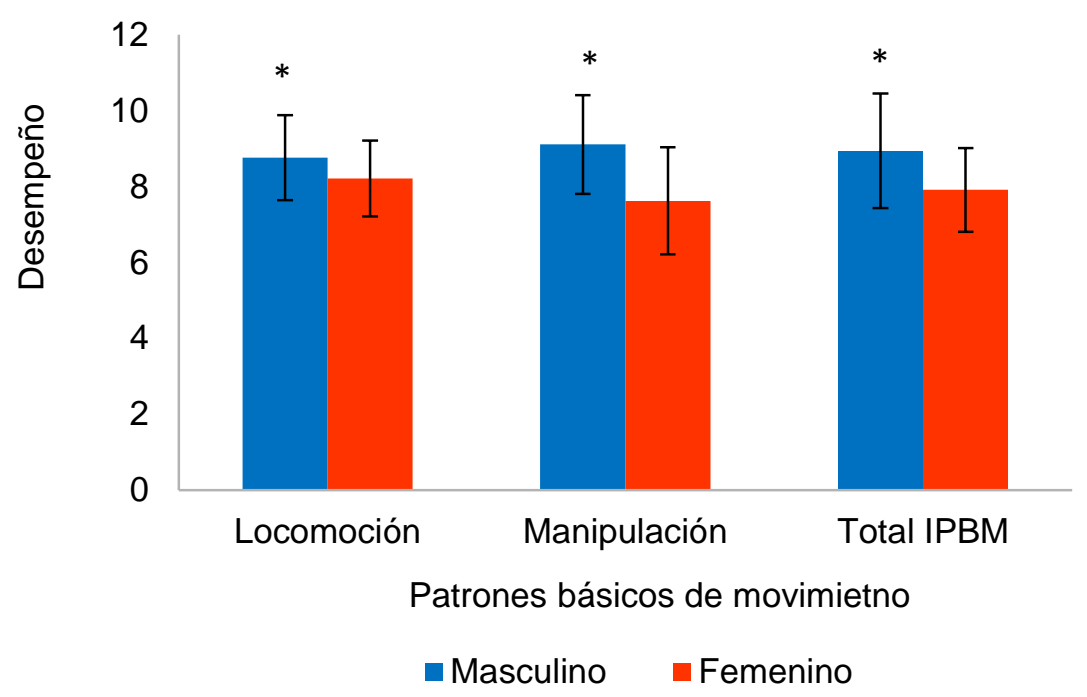

Figura 4. Desempeño para el puntaje total de la prueba, subescalas de locomoción y manipulación, por sexo. *Diferencias entre hombres y mujeres $(p<, 05)$. Fuente: elaboración propia.

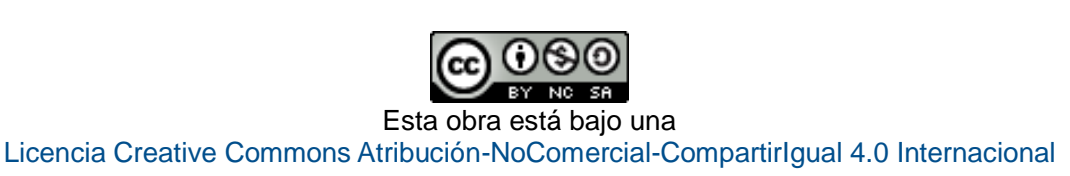




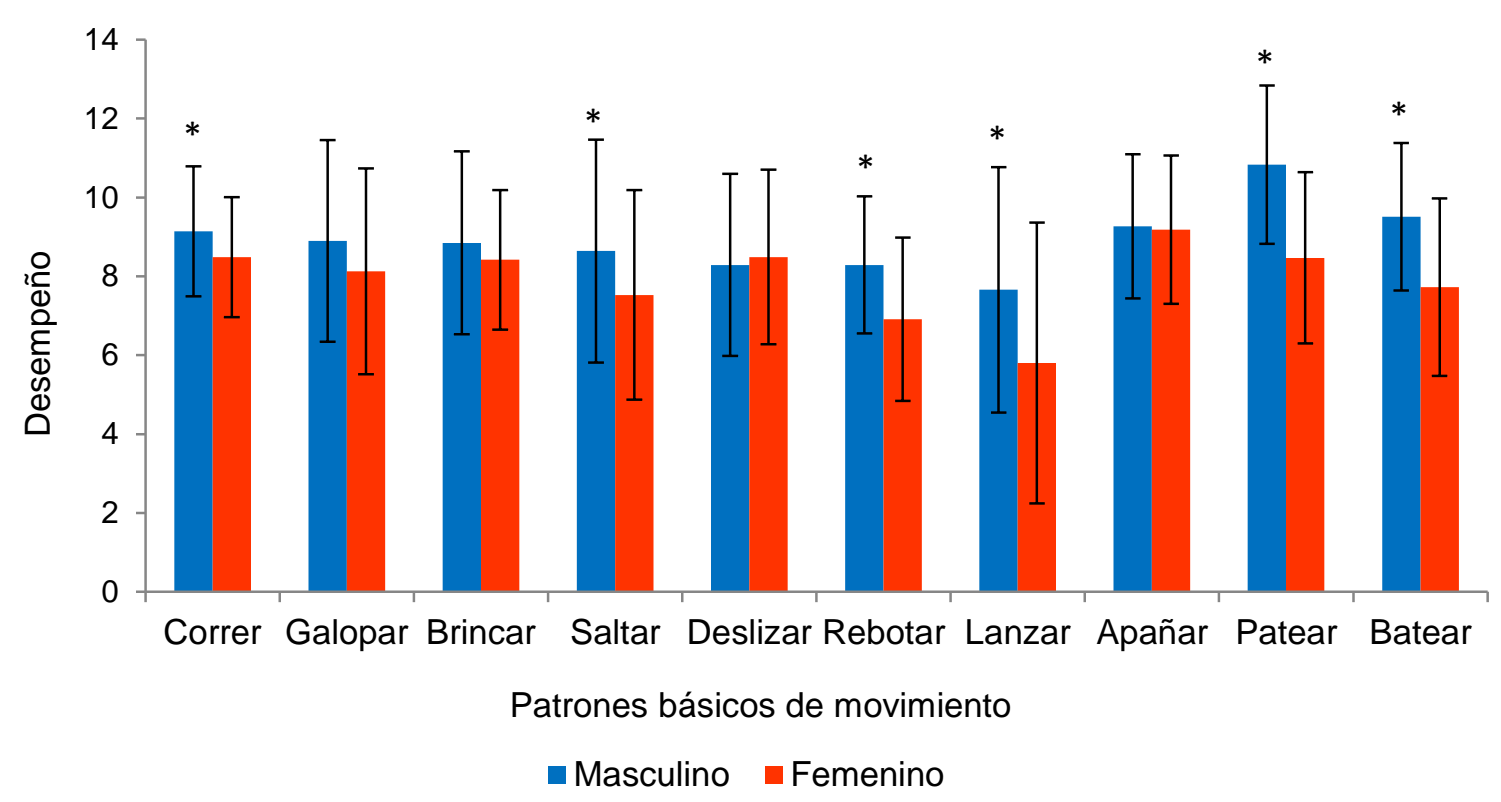

Figura 5. Desempeño para el puntaje de los patrones básicos de movimiento, por sexo. ${ }^{*}$ Diferencias entre hombres y mujeres $(p<, 05)$. Fuente: elaboración propia.

\section{DISCUSIÓN}

El propósito del presente estudio era examinar las diferencias de edad y sexo en el desempeño de los patrones básicos de movimiento en tres grupos de diferentes edades. Se esperaba un desempeño mejor en adolescentes y adultos-jóvenes en comparación con los infantes, sin embargo, los resultados no apoyaron dicha hipótesis para el puntaje total del IPBM y ambas subescalas (LM y OC). En relación con la segunda hipótesis, se encontró que los hombres presentaron un mejor desempeño que las mujeres para el total del IPBM y ambas subescalas (LM y OC).

En general, la mayoría de los participantes no lograron un desempeño eficiente en los PBM, esto se representa con un puntaje de 9 o menos. Los hombres del grupo de adolescentes presentaron el porcentaje de logro más alto, mientras que las niñas y las mujeres del grupo de adultos-jóvenes muestran el porcentaje más bajo. Tomando en cuenta estos resultados y la relación entre niveles de actividad física y desempeño motor, se puede creer que estos niveles de desempeño eficiente contribuyen a un estilo de vida

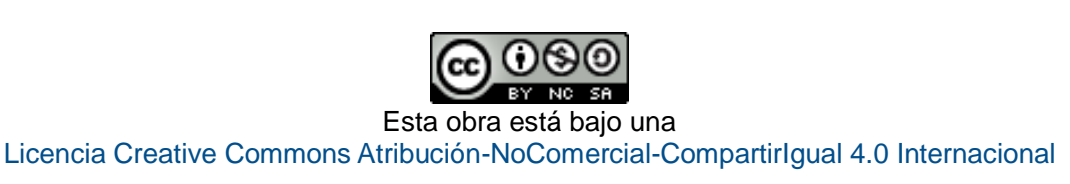


sedentario.

Para la primera hipótesis, se esperaba un desempeño mayor de los adolescentes y adultos-jóvenes en relación con los niños(as). Al evaluar el desempeño en PBM y en cada subescala, los resultados sugieren que el desempeño entre los grupos es similar y por debajo de un desempeño eficiente. Por lo tanto, se considera que la mayoría de los participantes no están practicando ni refinando los PBM en un ambiente de aprendizaje apropiado, o lo que sería peor, no están practicando estas destrezas del todo.

Al analizar cada destreza de forma individual, los adolescentes y adultos-jóvenes presentaron un mejor desempeño que los infantes en el patrón de galopar, y además solo en esta destreza los adultos-jóvenes alcanzaron un desempeño eficiente. Una tendencia similar encontraron Barnett et al. (2010) y Hardy et al. (2012) cuando evaluaron las destrezas locomotoras en infantes y adolescentes. Adicionalmente, en este estudio se encontró que todos los grupos de edades presentaron un desempeño eficiente en el patrón de patear y que los adultos-jóvenes presentaron un mejor desempeño que los adolescentes y los infantes. Por el contrario, Barnett et al. (2010) encontraron que patear tuvo un porcentaje bajo de mejoría de la etapa infantil a la adolescente, en comparación con destrezas como apañar y lanzar. Esto no es sorprendente si se considera que en Costa Rica el fútbol, que implica patear, es el deporte más popular. Aunque, es importante tomar en cuenta que la investigación de Barnett et al. se basó en una muestra longitudinal, mientras que estos resultados son de tipo transversal.

También, otra destreza que presentó diferencias entre grupos de edad fue lanzar por encima del hombro, ya que se encontró que los adultos-jóvenes mostraron un desempeño menor en comparación con infantes y adolescentes. Hardy et al. (2012) hallaron resultados similares, al evaluar apañar, patear y lanzar en niños(as) y adolescentes. Contrario a esto resultados, Lorson et al. (2013) encontraron que los adultos-jóvenes presentaron un mejor desempeño que los adolescentes. Una posible explicación de los resultados es que los infantes y los adolescentes en la actualidad tienen más oportunidades que los adultos-jóvenes cuando eran adolescentes o niños(as), por lo tanto muestran un mejor desempeño. Otra posible explicación, es que después de la adolescencia, los adultos-jóvenes dejan de practicar actividades que incluyen lanzar, por lo que puede haber una disminución en el desempeño debido a la falta de práctica.

Estos resultados apoyan la segunda hipótesis, las diferencias de sexo encontradas $-28-$

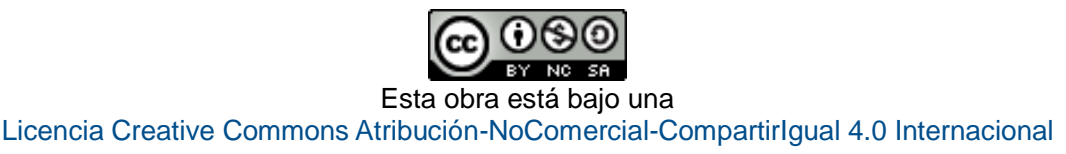


en el total de la prueba, las dos subescalas y en cinco de los diez patrones individuales evaluados son consistentes con otros estudios (Barnett et al., 2010; Hardy et al., 2012; Lorson et al., 2013; Spessato et al., 2012; Thomas \& French, 1985). El grupo de hombres presentó un mejor desempeño que las mujeres en correr, saltar, patear, batear y lanzar por encima del hombro. Similar a estos hallazgos Lorson et al. (2013) encontraron que los hombres y niños presentaron mejor desempeño que las mujeres y las niñas en el patrón de lanzar. También, Barnett et al. (2010) confirmaron que los niños presentaron un mejor desempeño que las niñas específicamente en los patrones manipulativos. Una posible explicación para las diferencias de sexo es que al utilizar instrumentos orientados al proceso en vez de instrumentos orientados al producto, las diferencias de sexo se le atribuyen a factores sociales en vez de a factores biológicos (Hardy et al., 2012).

El desarrollo de los PBM no es dependiente de la edad y no maduran de forma natural. La tarea, el individuo y el ambiente tienen un rol importante en el desempeño de las destrezas motrices (Gallahue \& Ozmun, 2005; Valentini \& Rudisill, 2004). Además, la etapa madura de desempeño se puede lograr con la práctica, instrucción y oportunidades de aprendizaje adecuados (Logan et al., 2012; Lubans et al., 2010; Valentini \& Rudisill, 2004). Por consiguiente, una posible explicación de los resultados encontrados es que los infantes, adolescentes y adultos-jóvenes no están recibiendo la práctica, instrucción o las oportunidades de aprendizaje necesarias para mejorar el desempeño de los PBM. Debido a estos resultados, es muy importante que los profesores de Educación Física incorporen práctica de los PBM, como lo propuso Carley (2010).

Al considerar que el desempeño de los PBM está relacionado con el nivel de actividad física y las capacidades físicas de las personas, además de estar relacionado negativamente con el índice de masa corporal (IMC) (Okely et al., 2004, 2001; Stodden et al., 2009; Stodden, True, Langendorfer, \& Gao, 2013); se puede especular que si se mejora el desempeño de los PBM, el nivel de actividad física y las capacidades físicas pueden mejorar, y el IMC puede disminuir. Las intervenciones motrices son estrategias efectivas para mejorar el desempeño de los PBM en infantes (Logan et al., 2012), pero poco se conoce si estas estrategias pueden ser efectivas en adolescentes y adultosjóvenes. Por tanto, es necesario desarrollar intervenciones que ayuden no solo a los niños(as), sino también a los adolescentes y adultos-jóvenes, para desarrollar un desempeño eficiente de los PBM. 
Se apoya la idea de que las personas se involucran en un estilo de vida físicamente activo por medio de uso de PBM en juegos, actividades deportivas y recreativas (Lubans et al., 2010; Robinson, 2011). Con base en los resultados, y considerando la limitación de un estudio transversal, se cree que un desempeño bajo en los PBM que se presenta en los diferentes grupos de edades, puede ser uno de los muchos factores que explican el incremento de los índices de sedentarismo. Por lo que, se concuerda que el desempeño en los PBM es un factor importante en las decisiones de una persona de ser físicamente activa. Además, es posible que los niveles bajos de actividad física, especialmente en la infancia, no promueven la suficiente práctica para alcanzar un desempeño eficiente de los PBM, como lo sugerido en Stodden, et al. (2008).

Al evaluar el proceso del desempeño (técnica) de los PBM, las diferencias de sexo se atribuyen principalmente a factores sociales: por lo que se recomienda a los profesores, entrenadores y profesionales del área, no subestimar a las niñas y mujeres por su sexo, y más bien tratar de alentarlas a practicar los PBM y mantenerse físicamente activas. Además, debido a los resultados, también se recomienda a todos los profesionales en salud a que se tomen su tiempo y enseñen PBM a todas las edades, y no asumir que por el hecho que ya no son niños(as), presentan un desempeño eficiente de los PBM.

\section{CONCLUSIÓN}

En conclusión, el desempeño similar que se encontró entre los grupos de edad en la mayoría de los PBM sugiere, que adolescentes y adultos-jóvenes no están mejorando o refinando su desempeño en las destrezas básicas y no están logrando un nivel de desempeño eficiente. Dada la relevancia de los PBM en los niveles de actividad física y en las capacidades relacionadas con salud, es importante que los profesionales en Educación Física y ciencias del movimiento humano y docentes a cargo de clases de Educación Física incluyan actividades para fortalecer el desempeño de los PBM en todas las edades, y ayudar a que los estudiantes desarrollen un desempeño eficiente. 


\section{REFERENCIAS}

Barnett, L. M., van Beurden, E., Morgan, P. J., Brooks, L. O., \& Beard, J. R. (2010). Gender differences in motor skill proficiency from childhood to adolescence: A longitudinal study. Research Quarterly for Exercise and Sport, 81(2), 162-170. Retrieved from http://www.tandfonline.com/doi/abs/10.1080/02701367.2010.10599663

Carley, M. (2010). The relationship between fundamental motor skill development and lifetime participation in physical activity. PSAHPERD, winter, 31-33.

Cohen, K. E., Morgan, P. J., Plotnikoff, R. C., Callister, R., \& Lubans, D. R. (2014). Fundamental movement skills and physical activity among children living in lowincome communities: a cross-sectional study. International Journal of Behavioral Nutrition and Physical Activity, 11(1), 49-58. http://dx.doi.org/10.1186/1479-5868-11-49

Gabbard, C. P. (2012). Lifelong Motor Development (6th ed.). San Francisco: BenjaminCummings Publishing Company. Retrieved from http://www.pearsonhighered.com/bookseller/product/Lifelong-MotorDevelopment/9780321734945.page

Gallahue, D. L., \& Ozmun, J. C. (2005). Understanding Motor Development: Infants, Children, Adolescents, Adults (6th ed.). McGraw-Hill Higher Education. Retrieved from http://trove.nla.gov.au/work/11623306

Goodway, J. D., \& Branta, C. F. (2003). Influence of a motor skill intervention on fundamental motor skill development of disadvantaged preschool children. Research Quarterly for Exercise and Sport, 74(1), 36-46. http://dx.doi.org/10.1080/02701367.2003.10609062

Hardy, L. L., Reinten-Reynolds, T., Espinel, P., Zask, A., \& Okely, A. D. (2012). Prevalence and correlates of low fundamental movement skill competency in children. Pediatrics, 130(2), e390-e398. http://dx.doi.org/10.1542/peds.2012-0345

Jiménez, J., Salazar, W., \& Morera, M. (2013). Diseño y validación de un instrumento para la evaluación de patrones básicos de movimiento. Motricidad. European Journal of Human Movement, 31(0), 87-97. Retrieved from http://www.eurjhm.com/index.php/eurihm/article/view/311

Logan, S. W., Robinson, L. E., Wilson, A. E., \& Lucas, W. A. (2012). Getting the fundamentals of movement: A meta-analysis of the effectiveness of motor skill interventions in children. Child: Care, Health and Development, 38(3), 305-315. http://dx.doi.org/10.1111/j.1365-2214.2011.01307.x 
Lorson, K. M., Stodden, D. F., Langendorfer, S. J., \& Goodway, J. D. (2013). Age and gender differences in adolescent and adult overarm throwing. Research Quarterly for Exercise and Sport, 84(2), 239-244. http://dx.doi.org/10.1080/02701367.2013.784841

Lubans, D. R., Morgan, P. J., Cliff, D. P., Barnett, L. M., \& Okely, A. D. (2010). Fundamental movement skills in children and adolescents. Sports Medicine, 40(12), 1019-1035. http://dx.doi.org/10.2165/11536850-000000000-00000

Okely, A. D., Booth, M. L., \& Chey, T. (2004). Relationships between body composition and fundamental movement skills among children and adolescents. Research Quarterly for Exercise and Sport, 75(3), 238-247. http://dx.doi.org/10.1080/02701367.2004.10609157

Okely, A. D., Booth, M. L., \& Patterson, J. W. (2001). Relationship of physical activity to fundamental movement skills among adolescents. Medicine and Science in Sports and Exercise, 33(11), 1899-1904. http://dx.doi.org/10.1097/00005768-200111000$\underline{00015}$

Robinson, L. E. (2011). The relationship between perceived physical competence and fundamental motor skills in preschool children. Child: Care, Health and Development, 37(4), 589-596. http://dx.doi.org/10.1111/j.1365-2214.2010.01187.x

Slotte, S., Sääkslahti, A., Metsämuuronen, J., \& Rintala, P. (2014). Fundamental movement skill proficiency and body composition measured by dual energy X-ray absorptiometry in eight-year-old children. Early Child Development and Care, 1-11. http://dx.doi.org/10.1080/03004430.2014.936428

Southall, J., Okely, A. D., \& Steele, J. (2004). Actual and perceived physical competence in overweight and nonoverweight children. Pediatric Exercise Science, 16(1), 15-24. Retrieved from http://journals.humankinetics.com/pes-backissues/PESVolume16Issue1February/ActualandPerceivedPhysicalCompetenceinOver weightandNonoverweightChildren

Spessato, B., Gabbard, C., \& Valentini, N. C. (2013). The role of motor competence and body mass index in children's activity levels in physical education classes. Journal of Teaching in Physical Education, 32(2), 118-130. Retrieved from http://journals.humankinetics.com/itpe-back-issues/itpe-volume-32-issue-2-april/therole-of-motor-competence-and-body-mass-index-in-childrenrsquos-activity-levels-inphysical-education-classes

Spessato, B., Gabbard, C., Valentini, N., \& Rudisill, M. (2012). Gender differences in -32 -

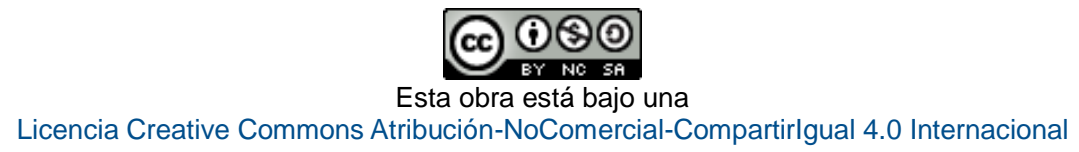


Brazilian children's fundamental movement skill performance. Early Child Development and Care, 183(7), 916-923. doi:10.1080/03004430.2012.689761

Stodden, D. F., \& Goodway, J. D. (2007). The dynamic association between motor skill development and physical activity. Journal of Physical Education, Recreation \& Dance, 78(8), 33-49. http://dx.doi.org/10.1080/07303084.2007.10598077

Stodden, D. F., Goodway, J. D., Langendorfer, S. J., Roberton, M. A., Rudisill, M. E., Garcia, C., \& Garcia, L. E. (2008). A developmental perspective on the role of motor skill competence in physical activity: An emergent relationship. Quest, 60(2), 290-306. http://dx.doi.org/10.1080/00336297.2008.10483582

Stodden, D. F., Langendorfer, S., \& Roberton, M. A. (2009). The association between motor skill competence and physical fitness in young adults. Research Quarterly for Exercise and Sport, 802), 223-229. http://dx.doi.org/10.1080/02701367.2009.10599556

Stodden, D. F., True, L. K., Langendorfer, S. J., \& Gao, Z. (2013). Associations among selected motor skills and health-related fitness: indirect evidence for Seefeldt's proficiency barrier in young adults? Research Quarterly for Exercise and Sport, 84(3), 397-403. http://dx.doi.org/10.1080/02701367.2013.814910

Valentini, N., \& Rudisill, M. E. (2004). Motivational climate, motor-skill development, and perceived competence: Two studies of developmentally delayed kindergarten children. Journal of Teaching in Physical Education, 23(3), 216-234. Retrieved from http://journals.humankinetics.com/itpe-backissues/JTPEVolume23Issue3July/MotivationalClimateMotorSkillDevelopmentandPerc eivedCompetenceTwoStudiesofDevelopmentallyDelayedKindergartenChildren

Wrotniak, B. H., Epstein, L. H., Dorn, J. M., Jones, K. E., \& Kondilis, V. A. (2006). The relationship between motor proficiency and physical activity in children. Pediatrics, 118(6), e1758-e1765. http://dx.doi.org/10.1542/peds.2006-0742

Participación: A- Financiamiento, B- Diseño del estudio, C- Recolección de datos, D- Análisis estadístico e interpretación de resultados, E- Preparación de manuscrito. 